\title{
Geostatistical Modeling of Environmental Sound Propagation
}

\author{
O. Baume, H. Wackernagel, B. Gauvreau, F. Junker, M. Bérengier \\ and J.-P. Chilès
}

\begin{abstract}
The atmospheric influence on sound propagation along the ground surface is a critical issue for estimating the noise impact of industrial plants or road networks. Indeed, sound refraction in the surface layer has a dramatic impact on the geographical acoustic exposure. Many analytical and numerical models and studies based on the laws of physics are available in scientific papers whereas very few works in statistical analysis have been attempted. However several important practical issues need to be considered. Among these, time and space representativity of "in situ" measurements, sampling design, influence of meteorological and ground parameters on acoustic exposure show to be a few challenges. They need to be investigated with statistical tools taking into account space and time autocorrelation.

A new protocol which includes ground impedance monitoring, spatial micrometeorological and acoustical characterization has been applied to an experimental campaign from June to August 2005 in a case of sound propagation from a point sound source on a grassy flat ground. The first geostatistical study on such a multivariable experimental database is presented. It adresses both the issue of modelling space varying impedance properties of an homogeneous meadow and the issue of modelling the acoustic field itself. This latter includes an analysis of the spatial variogram of the acoustic field residual calculated from a basic physical model as an external drift.
\end{abstract}

\section{Introduction}

Long Range Sound Propagation in the surface layer of the atmosphere is mainly influenced by the ground impedance, the ground topography and the micrometeorological conditions (see for instance Wiener and Keast (1959), Attenborough et al. (1980) or Rasmussen (1982)). In this field of research realistic numerical models derive from the linearized Euler's equations or the Helmholtz Equation

O. Baume

Department of Environmental Sciences, Wageningen University, The Netherlands

e-mail: olivier.baume@wur.nl 
(Ostashev, 1999). Particularly relevant results include the refraction of sound propagation in the form of vertical sound speed profiles and turbulence energy spectrum (Daigle et al., 1983; Wilson and Thomson, 1994; Dallois et al., 2001). But these advanced models have a heavy cost in CPU-time, especially when attempting to express the results of these models in more practical terms (1/3 octave bands e.g. or even more global noise level). Then the calculation time does not allow a time analysis of the influence of micro-meteorological conditions and ground characteristics on the environmental impact of a sound source.

On the other hand, the measurement of the noise impact of a road or an industrial plant is basically held with a poor space sampling design and a short time duration without in-situ representativity estimation. An estimate of the sound level is thus calculated with very empirical methods which do not take into account the total range of micro-meteorological conditions. New statistical work is needed to complete our knowledge for predicting in time and space the noise level emitted from a specific source. The geostatistical tools seem promisingly rich enough to support the needs of statistical modelling.

Laboratoire Central des Ponts et Chaussées, Électricité de France and the Geostatistics Group of the Centre de Géosciences (École des Mines de Paris) collaborate to develop new statistical methods for time and space estimation of the impact of a noise source. A new campaign with a space distribution of the sensors and a specific time and frequency sampling design which was set up by acousticians was held in 2005 from June to August. The database is proposed for a first geostatistical study in Long Range Sound Propagation.

First, we describe the protocol and focus on the data design. Second, the ground properties are studied at a day-time scale and a geostatistical model is proposed. The same variogram model is also used in conditional simulations. Third, we apply a universal kriging procedure to acoustic measurements. It includes the output of a simple physical model which gives a first order approximation of propagation of sound around a point source in a homogeneous field. Finally a linear drift component is included and it appears that it reflects the influence of the micro-meteorological conditions on the acoustic field.

\section{The Measurement Campaign}

\section{The Experimental Protocol}

Micro-meteorological towers have been used to determine the wind speed and temperature vertical gradients of the experimental site. These measurements will not be included into the geostatistical model but will be used separately for interpretation of the results.

\section{Acoustical Configuration}

In acoustics nothing is more sensitive to various micro-meteorological events on a flat ground than an omni-directional source. Its main advantage is to observe 
the time variability of acoustical time series which become very characteristic of weather conditions (Baume et al., 2005). Besides, its drawback is that its geometrical spreading for a constant signal imposes a fast decay in space and extraneous noise becomes tricky quite close to the source. The Brüel \& Kjaer 4296 omnidirectional source emitting a pink noise level worth $115 \mathrm{~dB}$ is located 2 meters above the ground. As shown on the left picture of Fig. 1, microphones were settled at 2 and 4 meter high along 3 main axes corresponding to sound propagation planes. The distance between 2 acoustical masts is $25 \mathrm{~m}$.

The acoustical sensors allow to record equivalent integrated sound levels (denoted $L_{e q}$ ) for periods greater than 1 second. A 10 to 15 minutes period is generally chosen because the average of corresponding micro-meteorological conditions are relevant to characterize mean refraction of the surface layer of the atmosphere. This was demonstrated in a former study using correspondence analysis (Séchet and Zouboff, 1995).

\section{Ground Effect}

Very special care was taken to characterize the ground properties during this campaign. Its influence on sound levels increases when propagation conditions become favorable between the source and the receivers. Indeed as sound energy is focused along the ground (downward curvature of the sound rays) more energy can be absorbed by a porous surface.

One monitoring station was designed to allow almost continuous data acquisition (every 4 hours) of the ground impedance at one specific point in space (emphasized on the right map of Fig. 1 by a black triangle). Furthermore a parallel campaign was held to explore the spatial variation of the ground impedance by performing measurements at 13 locations of the experimental area, which were repeated 13 times (13 days between early June and late August 2005). The global impedance protocol is drawn on Fig. 1 (right).

\section{Validation of the Database Used for the Spatial Analysis}

We want to stress the care that was taken in validating the database. For example the rainy and strong wind periods needed to be suppressed because of the microphones'sensitivity. Moreover an average acoustical spectrum of the source at several distances was determined in order to get rid of extraneous noise such as a few sound events that can appear anywhere (trains passing by in the valley, sparse road trafic, crickets). For acoustical data, the validation is applied to $1 / 3$ octave bands ranging from $50 \mathrm{~Hz}$ to $8 \mathrm{kHz}$ of 1 s integrated $L_{e q}$.

For the ground impedance database each measured value of the spatial campaign is an average of 10 successive measurements which avoids the largest errors that are to be made with this protocol. After the campaign, the impedance value is converted into a phenomenological parameter called the specific air flow resistivity by fitting a ground model called the Delany and Bazley model (Delany and Bazley, 1970). 

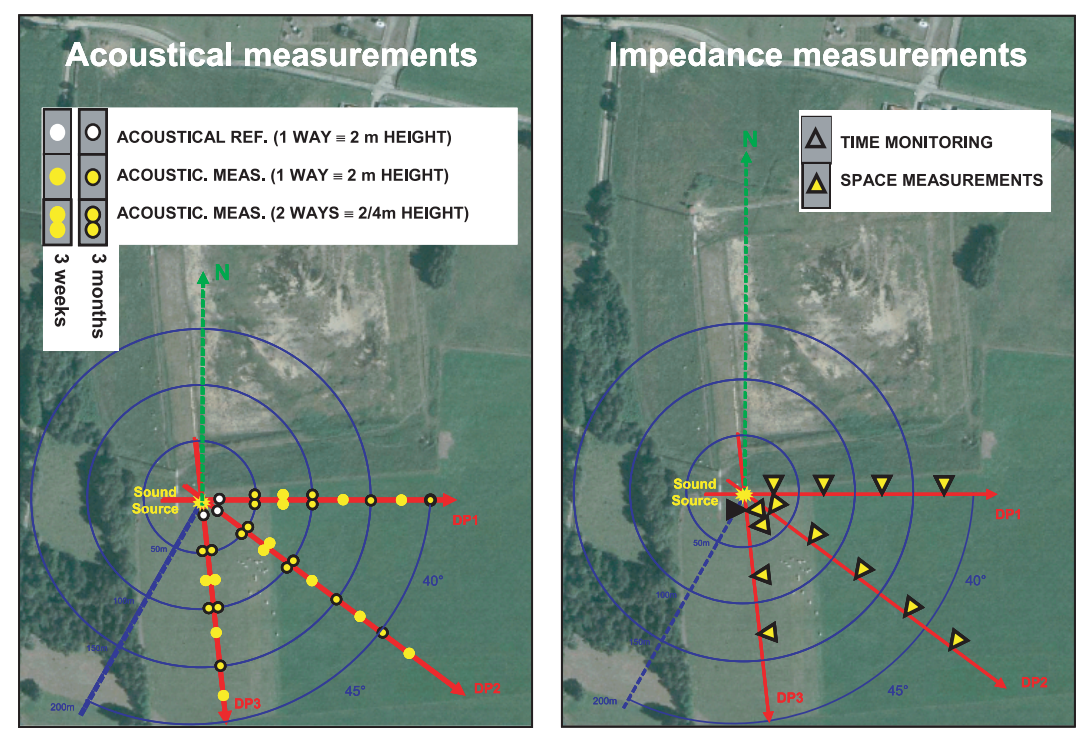

Fig. 1 Spatial distribution of acoustical (left) and ground impedance measurements (right) during the Lannemezan campaign in 2005

The specific air flow resistivity result is then obtained by averaging the Delany and Bazley model fit that was found by 3 different people. For this type of grassy ground, the best model to use is a 2 parameter model which includes the specific air flow resistivity (denoted $\sigma_{\text {ground }}$ ) and the thickness of the porous layer of the ground (denoted $e$ ) (Rasmussen, 1982).

More details on the validation process of the data during this campaign can be found in Junker et al. (2006).

\section{Data Analysis}

\section{Ground Properties Analysis}

The Time Monitoring measurement helped to provide evidence that the short period fluctuations are much smaller than the large fluctuations. The first is due to the day and night thermal gap and to the effect of the dew on the porous layer of the ground while the second is related to the general weather conditions. Thus we focused our analysis on the large time scaled evolution of the spatial measurements of $\sigma_{\text {ground }}$ along the 3 propagation axes DP1, DP2 and DP3. We first studied the autocorrelation of the data and then applied ordinary kriging and conditional simulations with the variogram models assuming second order stationarity of the random function.

The interest of kriging is to visualize the zones of the field where the ground is more reflective to sound energy (i.e. $\sigma_{\text {ground }}$ is higher) and those where the ground 
is more absorbing. The conditional simulations are useful in providing impedance fields as input data of numerical models of sound propagation.

\section{Modelling the Autocorrelation of the Specific Air Flow Resistivity Data}

As we had a fairly low number of samples there is quite some uncertainty about the true variogram of impedance. The experimental variogram of Fig. 2 illustrates how low the number of pairs is. Our fit of the variogram was guided by our knowledge of the experimental uncertainties.

From our experience on impedance measurements and the Delany and Bazley model we know that experimental uncertainties are about $20 \mathrm{kNsm}^{-4}$. This uncertainty shows as a measurement error in the ordinary kriging model which imposes a nugget effect worth 200 (semi-variance). See for instance the model we chose for June 20th at $14 \mathrm{~h} 00$ (Fig. 2(a)). The map of ordinary kriging performed with a unique neighborhood is shown on Fig. 2(b) and provides a smooth picture of the actual ground impedance. It represents a spatial variability that is greater than the uncertainties in the measurements. While the weather conditions evolved in such a way that the meadow dried from late June to late August, we found different variogram models - spherical, stable (power $3 / 2$ ) and cubic - with a range varying from $60 \mathrm{~m}$ to $180 \mathrm{~m}$. This precluded the use of a mean model over time.

\section{Use of Conditional Simulations}

Contrarily to kriging, conditional simulations do not provide a smooth picture of the spatial variability. Especially when a nugget effect is imposed in the variogram model, the simulated surfaces of the specific air flow resistivity is very erratic. As an example we show on Fig. 2(c) a corresponding conditional simulation of the model of June 20th. The gaussian assumption was made to simulate the target random function.

As the conditional simulations bring a realistic behavior of the parameter, they have an important application potential as input data for numerical models of Long Range Sound Propagation: these up to now did not have access to the spatial evolution of the ground properties. By repeated runs of the LRSP model with different members of a geostatistical simulation ensemble the sensitivity of sound to ground impedance can be assessed.

\section{Results and Discussion}

Air flow resistivity is the main parameter for characterizing the acoustical properties of a porous ground like a crop field, a meadow or even a field covered with snow. A complete measurement campaign is very demanding with respect to both human and material resources. The Lannemezan 2005 campaign is a step forward in the spatial characterization of environmental sound propagation. The experimental variograms resulted from a low number of measurements in space and were quite shaky. It was then possible to fit a variogram model which included a nugget effect to take into account measurement uncertainties. The resulting maps from ordinary kriging 


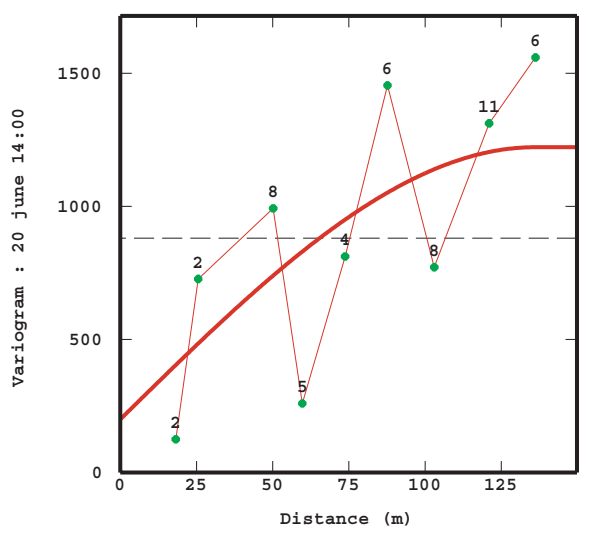

(a) Experimental variogram and variogram model
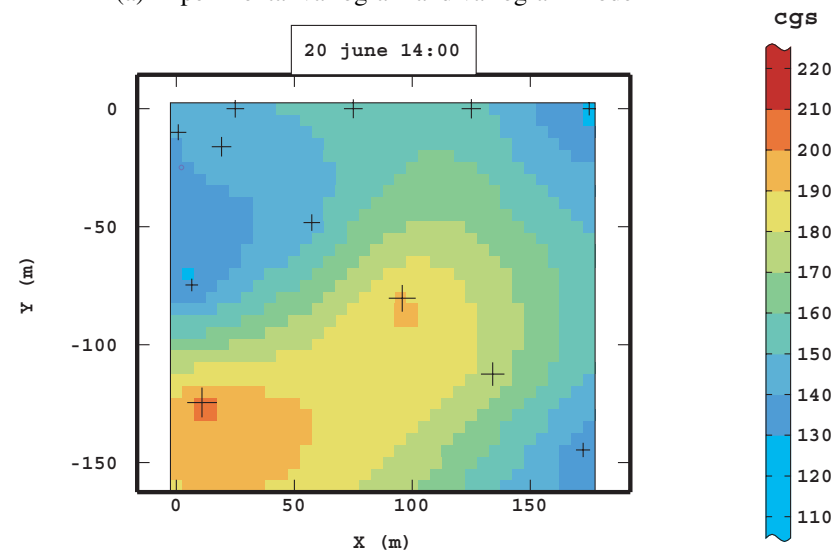

(b) Ordinary kriging estimation
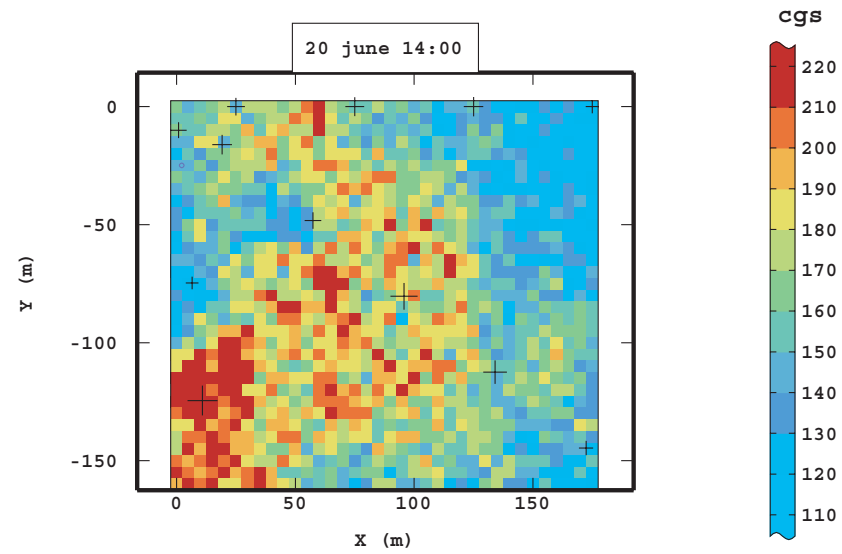

(c) Example of a conditional simulation

Fig. 2 Example: June 20th at 14:00 - nugget (sill 200) and spherical model (range $136 \mathrm{~m}$ and sill 1022); the number of pairs is written for each class of distance 
estimation give a new information about slow space variability of the specific air flow resistivity. The weather conditions have an impact on the zones which are more reflecting at the beginning of the campaign and then more absorbing as they get dryer and more porous.

The conditional simulations are ideal as a basis for sensitivity analyses of the influence of $\sigma_{\text {ground }}$ on acoustical results. Such a study should be an outcome of our work. In the next section a kriged mean value of the acoustic properties will be used (see details below).

\section{Sound Propagation Analysis}

This is to our knowledge the first study in geostatistical modelling of outdoor sound propagation. The purpose is to better understand the impact of different models on the interpolation for different $1 / 3$ octave bands and to give a first interpretation in terms of the ground and micro-meteorological influence on the acoustic propagation.

\section{Case Study}

To avoid the effects linked to the directivity of the source, the measured $L_{e q}$ (in $1 / 3$ octave bands) are transformed into attenuation levels between the reference microphones located 10 meters away from the source and more remote microphones (2 meter height) for each propagation direction (see Fig. 1). A particular day, June 22nd, is selected because many samples have complete spatial information. One 1/3 octave band is examined: $1 \mathrm{kHz}$ which is the central frequency in the log scale of the audible sounds.

\section{Universal Kriging}

Sound propagation is a non-stationary phenomenon which includes a geometrical spreading and atmospheric absorption. A simple first-order approximation explains the main component of the non-stationarity. So we orient the work to the development of a universal kriging procedure that includes a physical model in the external drift. Such an approach has already been used in air pollution modelling and is known in meteorology as data assimilation (see e.g. Wackernagel et al. (2004)). Besides, the external drift method is presented in Chilès and Delfiner (1999) and applied in other multivariate contexts in Wackernagel (2003).

The method can be split into 4 steps. The first step is the calculation of a first order physical model. The second step is a least squares fit of the model to the experimental data. We take 2 different options: one is a least squares fit without linear drift while the other includes a linear drift. The third step consists in modelling the residuals and the fourth includes the resulting variogram model to a kriging computation known as kriging with external drift. 


\section{Step 1: calculation of the first order physical model}

We propose to use the Embleton physical model (Embleton, 1983). It takes into account a mean spatial value of the specific air flow resistivity rather than a simulated field as it is used to calculate a first order approximation of the acoustic attenuation. The general form of the acoustic pressure above a porous half-space at location $\mathbf{x}$ is given by

$$
p(\mathbf{x})=p_{d}(\mathbf{x})+Q \cdot p_{r}(\mathbf{x}),
$$

where $p_{d}$ is the contribution of the real source, $p_{r}$ the contribution of the image source and $Q$ the complex form of the reflection coefficient of the porous ground. $Q$ explicitly depend on the impedance $Z_{\text {ground }}$. In the Embleton model, the Delany and Bazley approach is taken to express the impedance. The following is used

$$
Z_{\text {ground }}=Z_{0}\left(1+9.08\left(\frac{f}{\sigma_{\text {ground }}}\right)^{-0.754}+j 11.9\left(\frac{f}{\sigma_{\text {ground }}}\right)^{-0.732}\right)
$$

where $Z_{0}$ is the air impedance.

The relation 1 is frequency dependent and the computation is made for a minimum of 7 frequencies for a 1/3 octave band. The Fig. 3(a) displays a map of the output from this model which will subsequently be used as a drift component for the assimilation of the entire day of data. It shows an isotropic propagation around the omni-directional source located in the upper left corner.

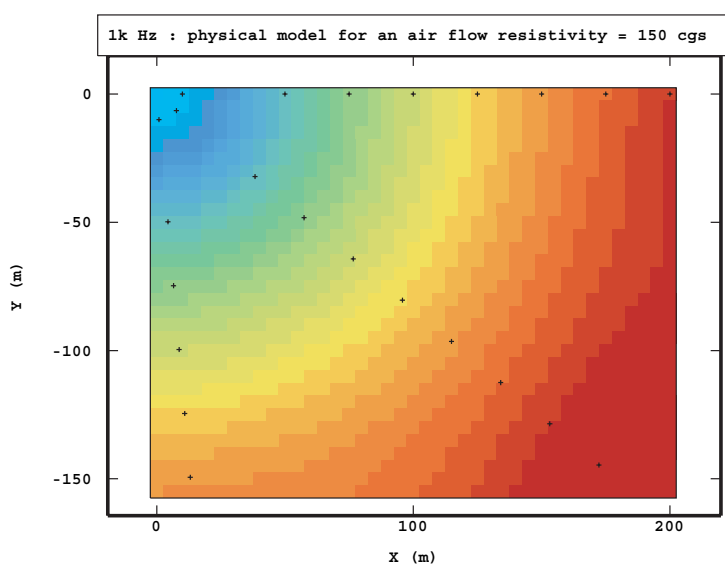

(a)
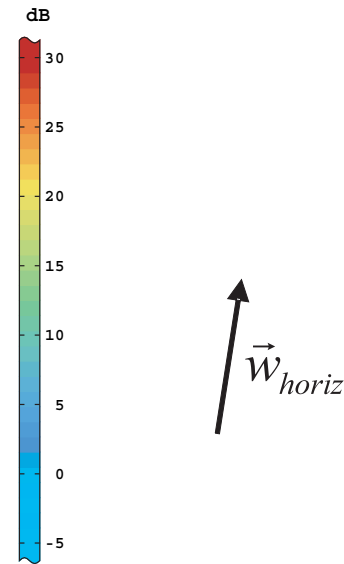

(b)

Fig. 3 (a) Acoustic attenuation calculated with the Embleton model for a mean $\sigma_{\text {ground }}=150 \mathrm{cgs}$ (b) Indicative horizontal wind direction for the sample June 22nd at 23:45 - wind speed at 10 meter high was $3.6 \mathrm{~m} . \mathrm{s}^{-1}$ 


\section{Step 2: least squares fit to the data}

The physical model output is fitted by least squares to the data at $23 \mathrm{~h} 45$ and the result is shown on Fig. 4(a). An option is to include also a linear drift component and the corresponding map is displayed on Fig. 4(b). The linear drift is an assumption made from the acousticians experience that the acoustic field is highly anisotropic following the wind direction (Wiener and Keast, 1959). We use this assumption to refine the method and then, the linear drift is calculated from acoustic measurements independently from wind data. In the least squares fit process example, the off-set component just lowers the attenuation whereas the linear drift gives an image of anisotropy due to the mean horizontal wind speed which was observed in the mean time (Fig. 3(b)).
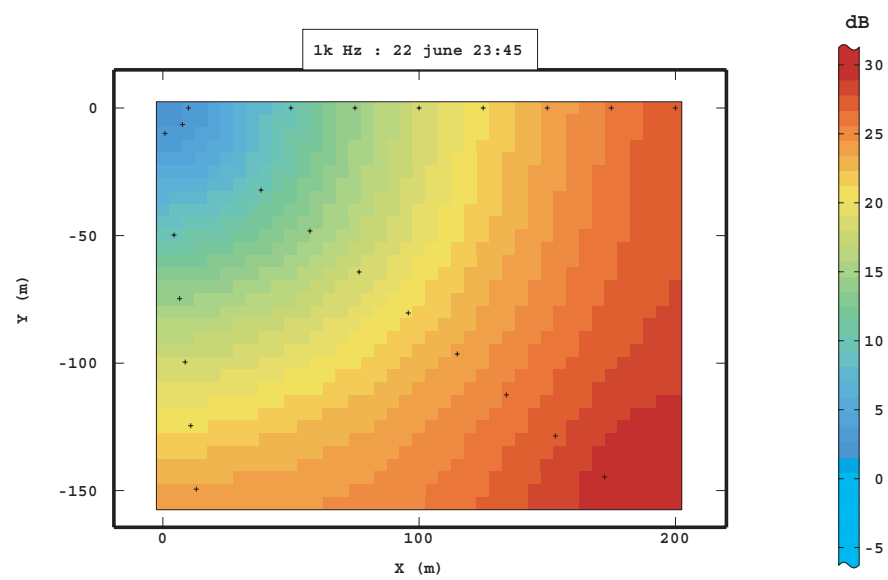

(a) Without linear drift

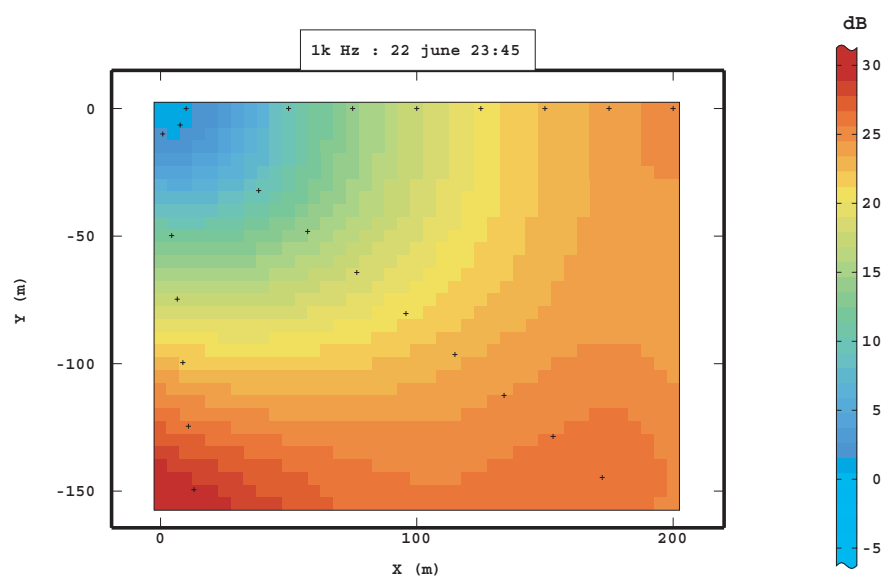

(b) Including a linear drift

Fig. 4 Least squares fit of the Embleton model to the measurements at $1 \mathrm{kHz}$, June 22nd at 23h45 


\section{Step 3: variograms of residuals}

The residuals between the measurements and the fitted drift (optionnally including a linear drift component) are computed and corresponding variograms are displayed on Fig. 5. The variogram of the residuals of the plain least-squares fit (Fig. 5(a)) shows a parabolic shape at the origin that cannot be reproduced well using a single cubic model. At large distances it does not stabilize at a sill, but continues to increase. The option of including a linear drift component to catch non-stationarity that is not explained by the Embleton model has the effect that the variance of the

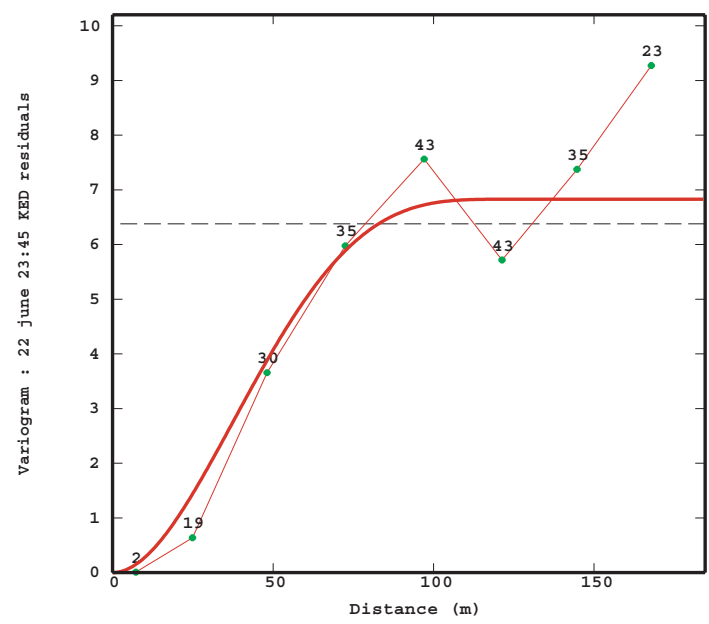

(a) Cubic model (range $125 \mathrm{~m}$ and sill 6.8).

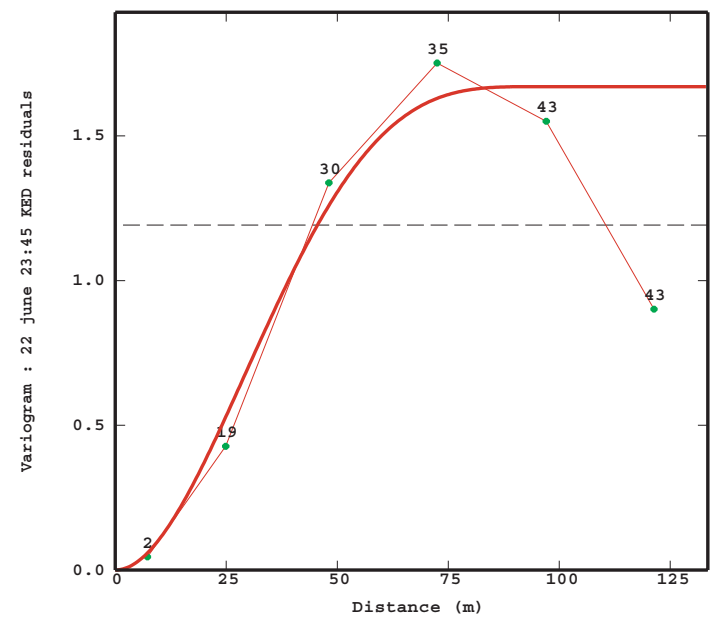

(b) Cubic model (range $96 \mathrm{~m}$ and sill 1.66).

Fig. 5 Variogram of residuals for least squares fit of external drift (a) without linear drift and (b) including a linear drift 
residuals is divided by a factor of 6 (Fig. 5(b)). Furthermore, the behavior of the variogram at the origin is fairly well compatible with a cubic model and the decrease beyond a distance of 75 is typical for the experimental variogram of residuals.

\section{Step 4: external drift kriging}

The external drift kriging was performed using optionally a linear drift component. The resulting maps are displayed on Fig. 6. The incorporation of a linear drift component strongly increases the anisotropy of the estimated attenuation with respect to the source in the top-left corner.
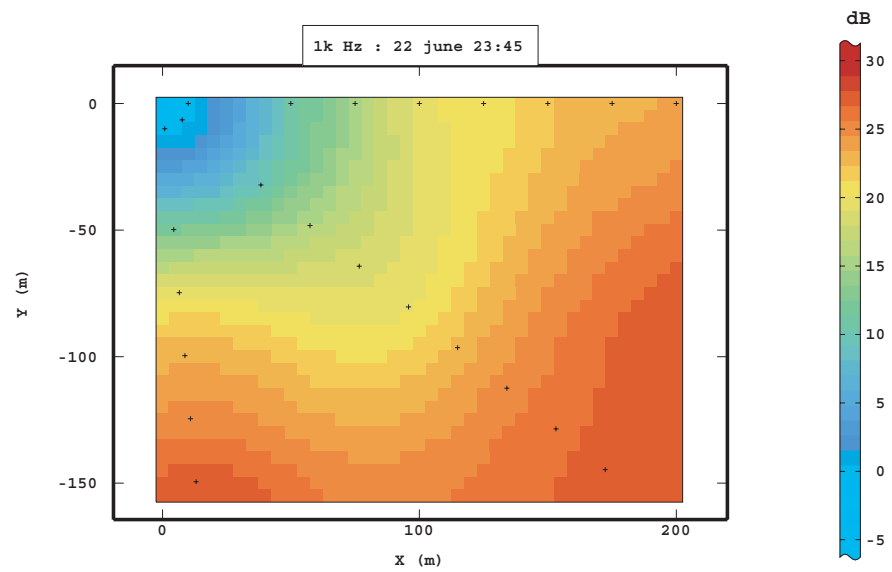

(a) Without linear drift.

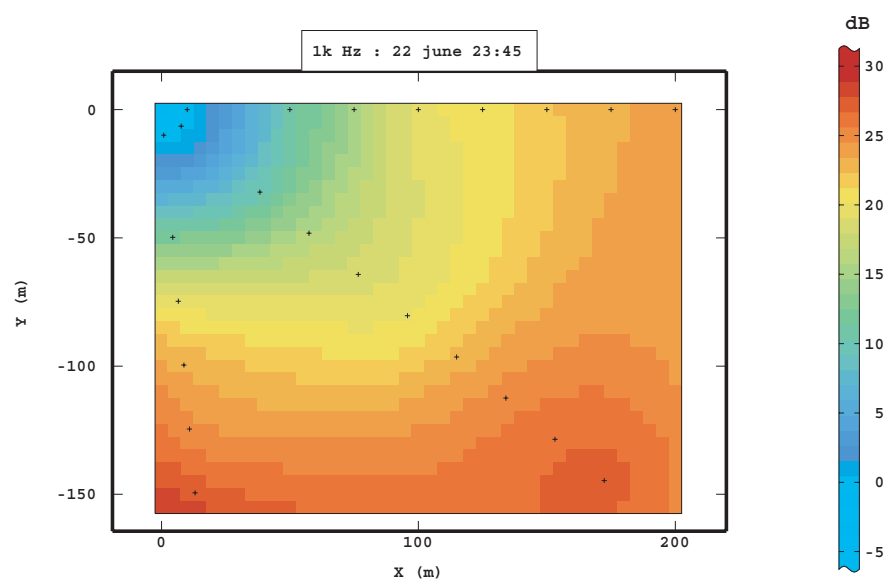

(b) Including a linear drift.

Fig. 6 Kriging with external drift 


\section{Results and Discussion}

The computations shown here for the measurements taken at $23 \mathrm{~h} 45$ were repeated for the whole day of June $22 \mathrm{nd}$ at $1 \mathrm{kHz}$. It turns out that the linear drift component obviously reflects the effect of the micro-meteorology on the sound propagation. In particular the wind direction and intensity seem to be related to the shape of the map of acoustic attenuation. In our example, the wind is strong enough to bring anisotropy in mean wind vector direction (South $\Rightarrow$ North direction parallel to the Y axis).

For low frequencies $(<500 \mathrm{~Hz})$, acoustical propagation is generally less influenced by micro-meteorology due to ground effects. So the inclusion of a linear drift to fit the Embleton model to the measurements has a slighter effect. On the contrary, at high frequencies (e.g. $1 \mathrm{kHz}$ ) a linear drift is needed to obtain a good anisotropy on the kriging map when the wind blows.

The residuals variogram is hardly ever interpretable in terms of physical phenomenon. Its shape should be related to the micro-meteorological turbulence for which autocorrelation has already been studied (Stull, 1988). The study of this relation requires another set of data with regularly spaced microphones.

\section{Conclusion and Outcomes}

The experimental data from impedance measurements during the Lannemezan 2005 campaign were analysed to obtain a succession of specific air flow resistivity maps with an ordinary kriging technique. The low number of data points in space let us use a nugget effect to model the experimental uncertainties. The resulting conditional simulations will give a first opportunity to use realistic field of $\sigma_{\text {ground }}$ as an input data of numerical models in Long Range Sound Propagation.

An entire day of acoustical data was studied for the first time with geostatistical tools. We developed a kriging procedure using an external drift. This drift was chosen to be a fit by least squares of a simple physical model which takes into account the unvarying phenomena of atmospheric sound propagation. When the wind conditions have a high order effect on sound propagation, the anisotropy must be modelled by the inclusion of a linear drift in the external drift. Then, the physical model is well reoriented and the residuals variogram stabilized to a sill. Finally, the output of the kriging calculation is more realistic.

We still have to determine which is the best strategy to use when the number of space measurements is too low to obtain the right linear drift. This lack of information must be compensated by the micro-meteorological data and further analysis on its correlation with the direction of the linear drift will be held.

Acknowledgments The authors of the present paper would like to acknowledge the French Ministry of Environment and Sustainable Development for its financial support to the Lannemezan 2005 experimental campaign. 


\section{References}

Attenborough K, Hayek SI, Lawther JM (1980) Propagation of sound over a porous half space. J Acous Soc Am 68(5):1493-1501

Baume O, Gauvreau B, Junker F, Wackernagel H, Bérengier M, Chilès J-P (2005) Statistical exploration of small scale variation in acoustic time series taking into account micro-meteorological conditions. Budapest, Hungary. Forum Acusticum

Chilès J-P, Delfiner P (1999) Geostatistics, modelling spatial uncertainty. Wiley series on probability and statistics. Wiley, New York

Daigle GA, Piercy JE, Embleton TFW (1983) Line-of-sight propagation through atmospheric turbulence near the ground. J Acoust Soc Am 74(5):1505-1513

Dallois L, Blanc-Benon P, Juvé D (2001) Long range sound propagation in a turbulent atmosphere within parabolic equation. Acta Acustica 87(6):659-669

Delany ME, Bazley EN (1970) Acoustical properties of fibrous absorbant materials. Applied Acoustics, 3

Embleton TFW (1983) Effective flow resistivity of ground surfaces determined by acoustical measurements. J Acoust Soc Am 74(4):1239-1244

Junker F, Gauvreau B, Cremezi-Charlet C, Gérault C, Ecotière D, Blanc-Benon P, Cotté B (2006) Classification de l'inuence relative des paramètres physiques affectant les conditions de propagation à grande distance : campagne expérimentale de lannemezan 2005. Tours, France. Congrès Francais d'Acoustique

Ostashev V (1999) Acoustics in Moving Inhomogeneous Media. E \& FN Spon

Rasmussen KB, (1982) A note on the calculation of sound propagation over impedance jumps and screens. Journal of Sound and Vibration 84(4):598-602

Séchet E, Zouboff V (1995) Application des méthodes factorielles à la caractérisation des effets météorologiques sur la propagation du bruit à grande distance. Bulletin de liaison Laboratoire des Ponts et Chaussées, 198(référence 3908)

Stull RB (1988) An introduction to boundary layer meteorology. Kluwer Academic, Dordrecht, The Netherlands

Wackernagel H (2003) Multivariate geostatistics, 3rd edn. Springer-Verlag, Berlin

Wackernagel H, Lajaunie C, Blond N, Roth C, Vautard R (2004) Geostatistical risk mapping with chemical transport model output and ozone station data. Ecological Model 179:177-185

Wiener FM, Keast DN (1959) Experimental study of sound over ground. J Acoust Soc Am, 31(6):724-733

Wilson DK, Thomson DW (1994) Acoustic propagation through anisotropic, surface layer turbulence. J Acoust Soc Am 96(2):1080-1095 